\title{
Multi-trait genetic evaluation for horn traits of economic importance in the Cape buffalo (Syncerus caffer caffer)
}

\author{
A.A. Lepori ${ }^{1}$, G.C. Josling ${ }^{1 \#}$, F.W.C. Neser ${ }^{1}$, P.C. Lubout ${ }^{2}$ \& J.B. van Wyk ${ }^{1}$ \\ ${ }^{1}$ University of the Free State, P.O. Box 339, Bloemfontein, 9300, South Africa \\ ${ }^{2}$ Wildlife Stud Services, Postnet Suite 489, Private Bag X025, Pretoria, South Africa
}

(Submitted 30 May 2018; Accepted 25 February2019; First published 25 April 2019)

\author{
Copyright resides with the authors in terms of the Creative Commons Attribution 4.0 South African License. \\ See: http://creativecommons.org/licenses/by/4.0/za \\ Condition of use: The user may copy, distribute, transmit and adapt the work, but must recognize the authors and \\ the South African Journal of Animal Science.
}

\begin{abstract}
The wildlife industry in South Africa has shown immense growth since the 1990s, which was brought about by the private game segment of the industry. In recent years, trophy quality Cape buffalo breeding animals have achieved extremely high prices. Much of the economic value of these animals can be attributed to horn size, which is important for breeding and hunting purposes. The main objective of the study was to estimate variance components for horn traits of economic importance as well as to develop guidelines for recording these traits. To date, no quantitative genetic analysis has been done for any traits in Cape buffalo. The total number of horn measurement records included in the evaluation was $\mathrm{n}=945$ for outer spread (BHSO), $n=470$ for tip to tip (BHTSCl), $n=468$ for left boss and $n=479$ for right boss. For descriptive statistics, males and females were considered separately while age was divided into clusters of six months. A multi-trait animal model using Monte Carlo Markov Chains methods was used for the estimation of genetic parameters. Results suggest that it is not economically viable to measure horn spread and tip to tip of females after 48 months of age. Horns of the males continue to grow beyond 91 months of age. Boss records were unreliable owing to the applied measurement techniques for female and young animals. An inbreeding coefficient of 0.008 was calculated, suggesting adequate genetic diversity in the studied population. The heritability estimates of the horn traits were low, showing that extreme care has to be taken to develop effective selection programmes for the buffalo game industry using their horn genetic parameters. Further quantitative studies are required to support the results of the current study.
\end{abstract}

Keywords: genetic correlation, genetic parameters, heritability, Monte Carlo Markov Chain

\# Corresponding author: buitendachgc@ufs.ac.za

\section{Introduction}

South Africa is one of the few countries in Africa to see an increase in wildlife numbers over the past three decades. During 2016, the number of herbivores on private land was estimated to be close to 6 million animals, while the total number of wild mammals across South Africa (including private and public land) is reportedly between 16 and 20 million animals (Taylor et al., 2015). The basis for the growth was provided by The Game Theft Act No. 105 of 1991 which allows private ownership of wildlife, giving game commercial value and creating alternative uses for agricultural land (Simpson, 2012).

The African buffalo forms part of the "Big Five" of hunting. Recently, trophy quality Cape buffalo breeding animals have achieved extremely high prices and exceptional individual animals can even reach millions of dollars at auction. Economic contribution of the live game trade (all species) and related activities is predicted to be close to $\$ 2$ billion per annum, based on 2014 data (Cloete et al., 2015). As the third highest income-earner for trophy hunting, buffalo also contribute significantly to the economics of the South African hunting industry with a predicted value of $\pm \$ 0.7$ billion during 2015 (Cloete et al., 2015).

Much of the economic value of buffalo can be attributed to horn size. Therefore, factors affecting the size of the horns are of particular importance to breeders. In turn, data recording of not only horn size, but also pedigree and reproduction information has become an integral part of buffalo management. Both male and female buffalo have horns, but a characteristic feature of adult bulls is the fusion of their horn bases, 
forming a continuous bone shield referred to as a "boss." From the base, the horns curve downwards before smoothly curving upwards and inwards. In cows, the horns are generally smaller, and the boss is less prominent (Alden et al., 1995). Horns of buffalo are classified and measured according to the boss-type horn measuring methods of the Rowland Ward (RW) and Safari Club International (SCl) trophy hunting systems (Van Rooyen et al., 2016). These systems have also been adopted to measure and evaluate the horn dimensions of live animals according to age.

Molecular genetic studies have been conducted on Cape buffalo to develop DNA markers for parentage verification (Greyling et al., 2008) and evaluation of genetic diversity of privately owned as well as free-roaming buffalo populations (Greyling, 2007; Van der Westhuizen et al., 2016). The aim of this study was therefore to (i) provide descriptive statistics, (ii) determine genetic parameters based on genomic verified pedigrees, and (iii) develop guidelines for recording horn traits of economic importance of Cape buffalo.

\section{Materials and Methods}

Field data on horn traits as well as genomic verified pedigree records of Cape buffalo were extracted from the complete data set (1020 animals from 35 private buffalo herds across South Africa from April 2003 to July 2016) provided by Wildlife Stud Services. Horn traits were measured according to both the RW and $\mathrm{SCl}$ systems. Figure 1 illustrates the measuring techniques of the economically important horn traits considered in the present study. The $\mathrm{SCl}$ horn traits included the tip to tip (BHTSCl), left (BOSL), and right (BOSR) boss width measurements, while the outer spread (BHSO) was considered in the RW system.
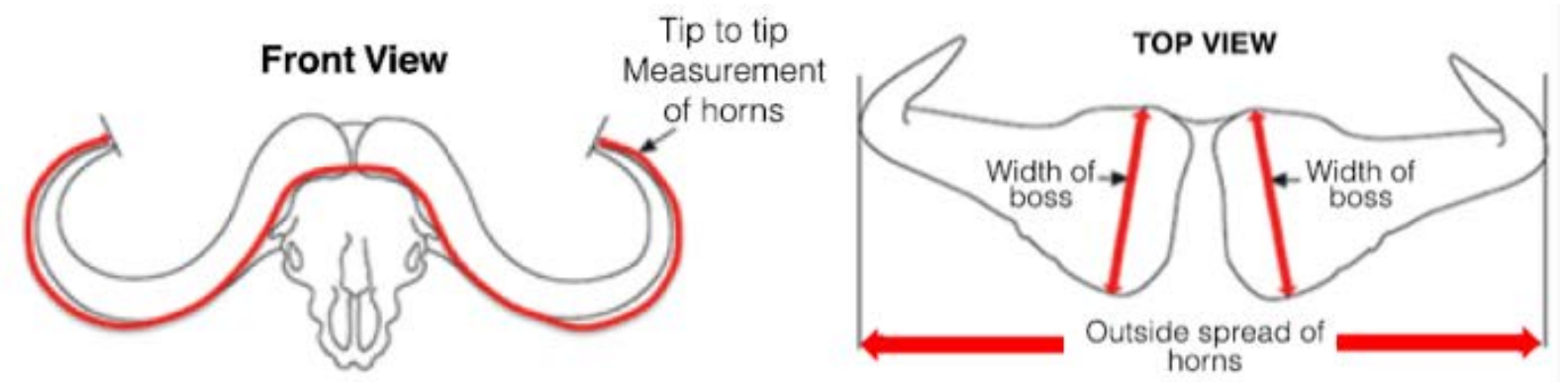

Figure 1 Horn measuring techniques of African buffalo according to the SCl (Tip to tip; left and right boss widths) and RW (outside spread) trophy systems

The tip to tip measuring technique involves starting at one horn tip and following the outermost curve, measuring in a straight line over the gap between the horns of the forehead and continuing to follow the curve to the tip of the other horn in one measurement. Left and right boss widths are measured respectively at the widest points of the horn bases. The outer points of the horn curves represent the outside spread of the horns (Van Rooyen et al., 2016).

The first dataset was filtered using R statistical computing software (R Core Team, 2017), eliminating animals without date of birth and outliers deviating more than four standard deviations from the mean within age clusters. The total number of records included in the evaluation were $n=945$ for BHSO, $n=470$ for BHTSCl, $n=468$ for BOSL, and $n=479$ for BOSR. For the descriptive statistics males and females were considered separately while age was divided into clusters of six months.

For the estimation of genetic parameters, a multi-trait animal model with repeated measures was assessed, using Markov chain Monte Carlo procedures (MCMC) under the R software package MCMCglmm (Hadfield, 2010). Prior distributions included for direct, permanent and residual variances were relatively uninformative, emulating an inverse gamma distribution with a variance of 1 and a degree of belief parameter of 0.002, often used for variance components (Hadfield, 2017). The MCMC analysis was set up with a total number of 200,000 iterations with 20,000 iterations used for the burn-in process, while one in every five iterations were stored in memory. Convergence was assessed using the Cramer-von-Mises statistic test.

Expanded from Mrode (2006), in matrix notation the multi-trait animal model can be represented as: 


$$
\left[\begin{array}{c}
y_{1} \\
y_{2} \\
\vdots \\
y_{n}
\end{array}\right]=\left[\begin{array}{cccc}
X_{1} & 0 & 0 & 0 \\
0 & X_{2} & \cdots & 0 \\
\vdots & \vdots & \ddots & \vdots \\
0 & 0 & \cdots & X_{n}
\end{array}\right]\left[\begin{array}{c}
b_{1} \\
b_{2} \\
\vdots \\
b_{n}
\end{array}\right]+\left[\begin{array}{cccc}
Z_{1} & 0 & 0 & 0 \\
0 & Z_{2} & \cdots & 0 \\
\vdots & \vdots & \ddots & \vdots \\
0 & 0 & \cdots & Z_{n}
\end{array}\right]\left[\begin{array}{c}
a_{1} \\
a_{2} \\
\vdots \\
a_{n}
\end{array}\right]+\left[\begin{array}{cccc}
S_{1} & 0 & 0 & 0 \\
0 & S_{2} & \cdots & 0 \\
\vdots & \vdots & \ddots & \vdots \\
0 & 0 & \cdots & S_{n}
\end{array}\right]\left[\begin{array}{c}
p e_{1} \\
p e_{2} \\
\vdots \\
p e_{n}
\end{array}\right]+\left[\begin{array}{c}
e_{1} \\
e_{2} \\
\vdots \\
e_{n}
\end{array}\right]
$$

where: $y_{i}$ is a vector that represents the observed phenotypic records of the ith trait (from 1 to $\mathrm{n}$ ),

$X_{i}$ is an incidence matrix of fixed effects associated with trait $i$,

$b_{i}$ is a vector of the regression coefficients for the fixed effects of each trait $i$,

$Z_{i}$ is an incidence matrix relating traits $i$ records to animals in the pedigree,

$a_{i}$ is the vector of additive random animal effects for trait $i$,

$S_{i}$ is an incidence matrix of the permanent environmental effects,

$p e_{i}$ is the vector of permanent environmental effects for trait $i$, and

$e_{i}$ is a vector of trait's $\mathrm{i}$ random residual values (not considered by the fixed and other random effects).

The evaluation of random effects assumed that each value was normally distributed and independent.

$\operatorname{var}\left[\begin{array}{c}a_{1} \\ \vdots \\ a_{n} \\ q_{1} \\ \vdots \\ q \\ e_{1} \\ \vdots \\ e_{n}\end{array}\right]=\left[\begin{array}{ccc}\mathbf{G} & 0 & 0 \\ 0 & \mathbf{Q} & 0 \\ 0 & 0 & \mathbf{R}\end{array}\right]$

$\mathbf{G}, \mathbf{Q}$ and $\mathbf{R}$ being (co) variance matrices of genetic, permanent environmental and residual effects represented as:

$$
\begin{aligned}
& \operatorname{var}\left[\begin{array}{c}
a_{1} \\
\vdots \\
a_{n}
\end{array}\right]=\left[\begin{array}{ccc}
A \sigma_{a_{i}}^{2} & \cdots & A \sigma_{a_{i n}} \\
\vdots & \ddots & \vdots \\
A \sigma_{a_{i n}} & \cdots & A \sigma_{a_{n}}^{2}
\end{array}\right]=\mathbf{G}, \\
& \operatorname{var}\left[\begin{array}{c}
p e_{1} \\
\vdots \\
p e_{n}
\end{array}\right]=\left[\begin{array}{ccc}
I \sigma_{q_{i}}^{2} & \cdots & I \sigma_{q_{i n}} \\
\vdots & \ddots & \vdots \\
I \sigma_{q_{i n}} & \cdots & I \sigma_{q_{n}}^{2}
\end{array}\right]=\mathbf{Q}, \text { and } \\
& \operatorname{var}\left[\begin{array}{c}
e_{1} \\
\vdots \\
e_{n}
\end{array}\right]=\left[\begin{array}{ccc}
I \sigma_{e_{i}}^{2} & \cdots & I \sigma_{e_{i n}} \\
\vdots & \ddots & \vdots \\
I \sigma_{e_{i n}} & \cdots & I \sigma_{e_{n}}^{2}
\end{array}\right]=\mathbf{R}
\end{aligned}
$$

where: $\mathbf{I}$ is an identity matrix with its dimensions equalling the total number of evaluated animals,

A is the pedigree-based relationship matrix, and

the diagonal elements ${\sigma_{a_{i}}}^{2}, \sigma_{p e_{i}}{ }^{2}$ and ${\sigma_{e}}_{e_{i}}{ }^{2}$ are the genetic, permanent environmental and residual effects variances for traits $i$ to $n$, having $\sigma_{a_{i n}}, \sigma_{p e_{i n}}$ and $\sigma_{e_{i n}}$ as their corresponding covariances.

The definitive animal models for each trait evaluated, were:

$$
y_{i j k l m o p}=\mu+\operatorname{sex}_{i}+a g e_{j}+c s_{k}+o s_{l}+c g_{m}+a_{o}+p e_{p}+e_{i j k l m o p}
$$

where: $y_{i j k l m o p}$ referring to the $i j k l m o p^{\text {th }}$ observation of an animal in the $i j k l m o p^{\text {th }}$ subclass, $\mu$ corresponds to the trait's population mean,

$\operatorname{sex}_{i}$ denominates the $i^{\text {th }}$ effect of sex,

$a g e_{j}$ is the $j^{\text {th }}$ effect of age in months when the animal was measured, 


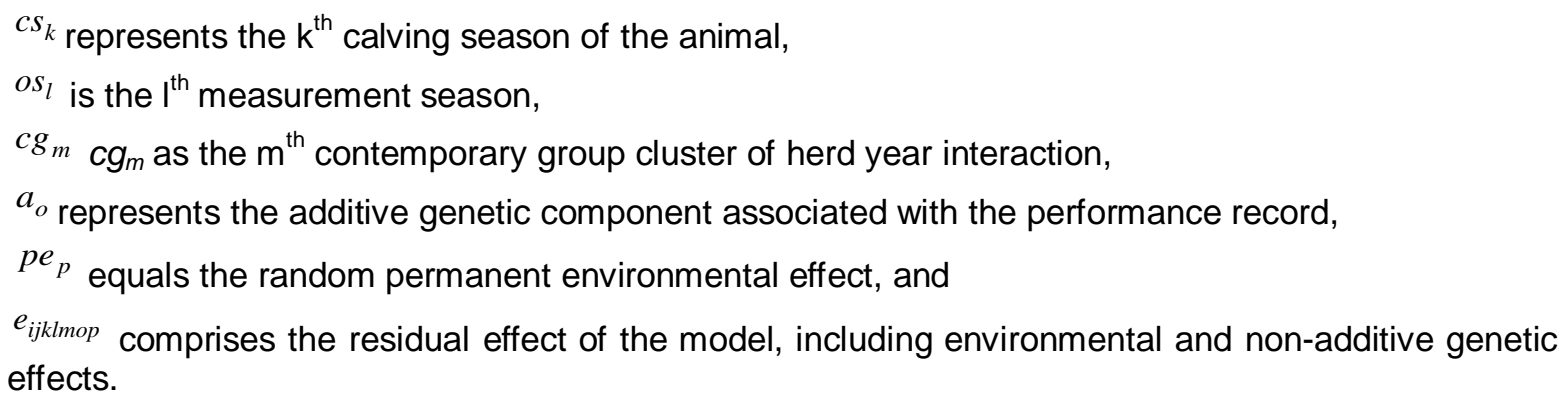

Following Falconer and Mackay (1996), estimated heritability estimates $\left(h^{2}\right)$ were derived using the average of each individual posterior distribution heritability, as:

$$
h^{2}=\frac{\sigma_{a}^{2}}{\sigma_{a}^{2}+\sigma_{p e}^{2}+\sigma_{e}^{2}}
$$

where: $\sigma_{a}^{2}$ being the additive genetic variance,

$\sigma_{p e}^{2}$ the permanent environmental variance, and

$\sigma_{e}^{2}$ the residual variance.

The posterior genetic correlations $r_{x y}$ were calculated as displayed by Wilson et al. (2010), for the average of each posterior distribution correlation described as:

$$
r_{x y}=\frac{\sigma_{x y}}{\sigma_{x} \times \sigma_{y}}
$$

where: $\sigma_{x y}$ is the posterior genetic covariance between traits $x$ and $y$, and

with the posterior genetic standard deviations of traits $\mathrm{x}$ and $\mathrm{y}$ being $\sigma_{x}$ and $\sigma_{y}$ respectively.

\section{Results and Discussion}

The results presented in Table 1 illustrate that the sex effect was highly significant $(P<0.05)$ within all the traits. An in-depth illustration of the information summarised in Table 1 is shown in Figures $2-4$ which also demonstrates the differences in age.

Table 1 Descriptive statistics for male and female buffalo horn traits demonstrating the mean, minimum

\begin{tabular}{|c|c|c|c|c|c|c|c|c|}
\hline & \multicolumn{4}{|c|}{ Male } & \multicolumn{4}{|c|}{ Female } \\
\hline & BHSO & BHTSCI & BOSL & BOSR & BHSO & BHTSCI & BOSL & BOSR \\
\hline Mean $^{a}$ & 29.93 & 70.16 & 12.97 & 12.96 & 26.04 & 61.01 & 7.95 & 8.02 \\
\hline Min. & 10.00 & 34.00 & 4.25 & 4.25 & 10.88 & 23.50 & 5.00 & 5.00 \\
\hline Max. & 53.00 & 100.50 & 21.00 & 21.00 & 38.75 & 78.50 & 12.00 & 12.00 \\
\hline
\end{tabular}
(min.) and maximum (max.) values for BHSO, BHTSCI, BOSL and BOSR in inches

${ }^{\mathrm{a}}$ indicates highly significant differences between male and females for all traits

$\mathrm{BHSO}=$ Horn spread; $\mathrm{BHTSCI}=$ Tip to tip index; BOSL = boss left measurement; BOSR = boss right measurement

Figures 2 and 3 (A) suggests that the horns of the male continue to grow up to 96 months of age in terms of the spread and tip to tip dimensions. On the other hand, the studied female population shows that 
horn growth reaches a plateau between 43 and 48 months of age (Figures 2-B and 3-B). Based on these results, it is not economically viable to measure the horn spread and tip to tip of females after the age of 43 months. However, further studies for the development of growth curves using repeated measurements for the same animals throughout their lifetime are required to confirm results of the current study.
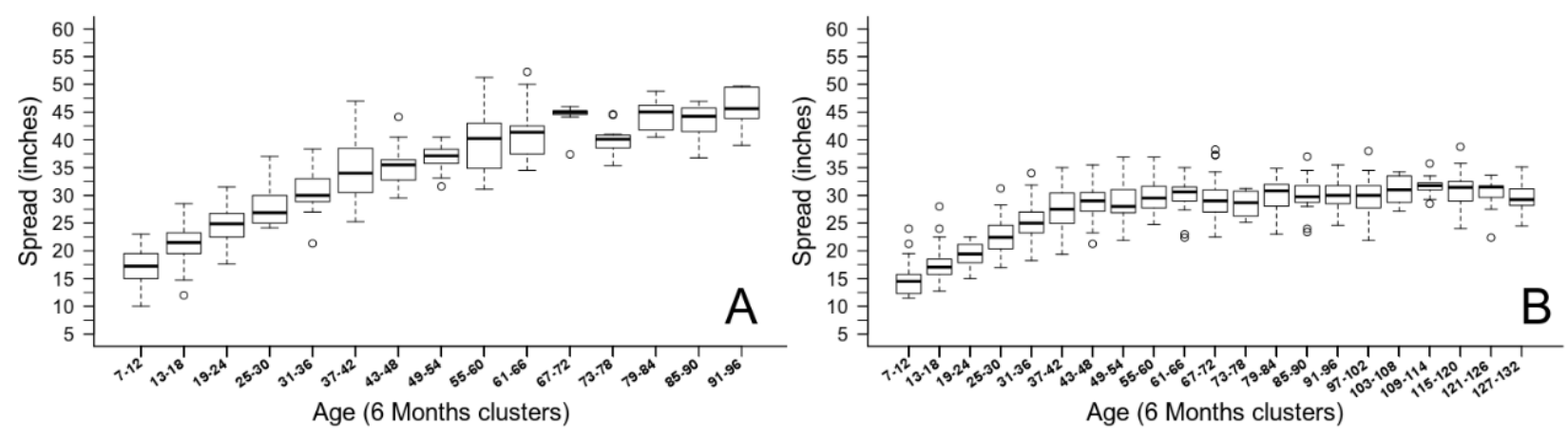

Figure 2 Horn spread distribution (BHSO) for male (A) and female (B) clustered within 6 months
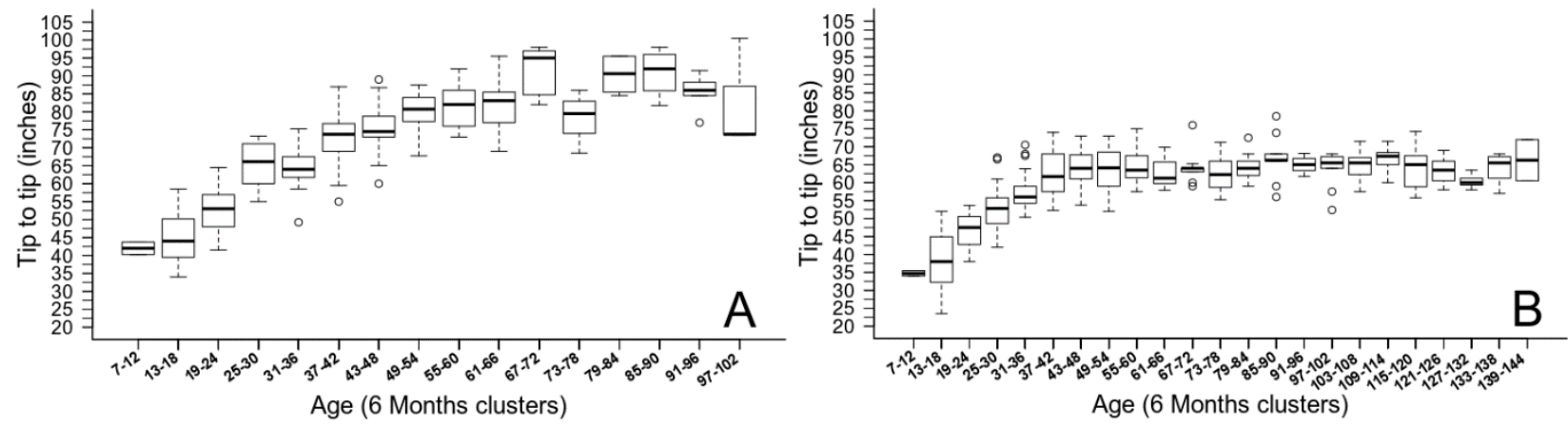

Figure 3 Horn tip to tip distribution (BHTSCI) for male (A) and female (B) clustered within 6 months

The start and end points of the boss measurements are not as clearly defined in young animals and females compared to sub-adult and mature males. Factors affecting the accuracy of the boss measurements of young animals between 7 - 12 months and females in general are unclear, but the measuring technique itself might contribute to these errors as demonstrated in Figure 4. For females (B and D) not much variation between ages from 37 - 42 months onwards can be detected, suggesting horn boss growth in females is not evident. Boss size of males reach a peak at 67 - 72 months of age and then show a slight downward trend thereafter. This decrease is thought to be due to a loss in volume as a result of the hardening of the boss. Based on the similarities of the left and right boss it indicates that only one side is often measured and the other is assumed to be equal. Therefore, it is inaccurate to compare differences between the left and right boss at this stage.

According to their posterior standard deviations (SD) (Table 2), variance components for BHSO were significant; however, the additive variance for $\mathrm{BHTSCl}$ and the boss measurements were not significant. The permanent environmental and residual variance estimates for these traits were nevertheless significant.

Heritability estimates (Table 2) were found to be low (under 0.2), and with a low accuracy of estimation based on their SD (except for BHSO). These results are lower compared to those presented by Lepori et al. (2018) and below the literature values of horn traits for wild Soay and bighorn sheep, which ranged from 0.223 - 0.442 (Poissant et al., 2008; Johnston et al., 2009; Pigeon et al., 2016). Therefore, due to the small sample sizes and/or protocol recording issues, it is suggested that more data, especially the number of repeated records, and further studies are required to confirm these results. It is also important to note that the use of MCMC procedures to analyse the data have improved the accuracy of prediction of the genetic parameters in comparison with what Lepori et al. (2018) have reported when using Al-REML procedures. 

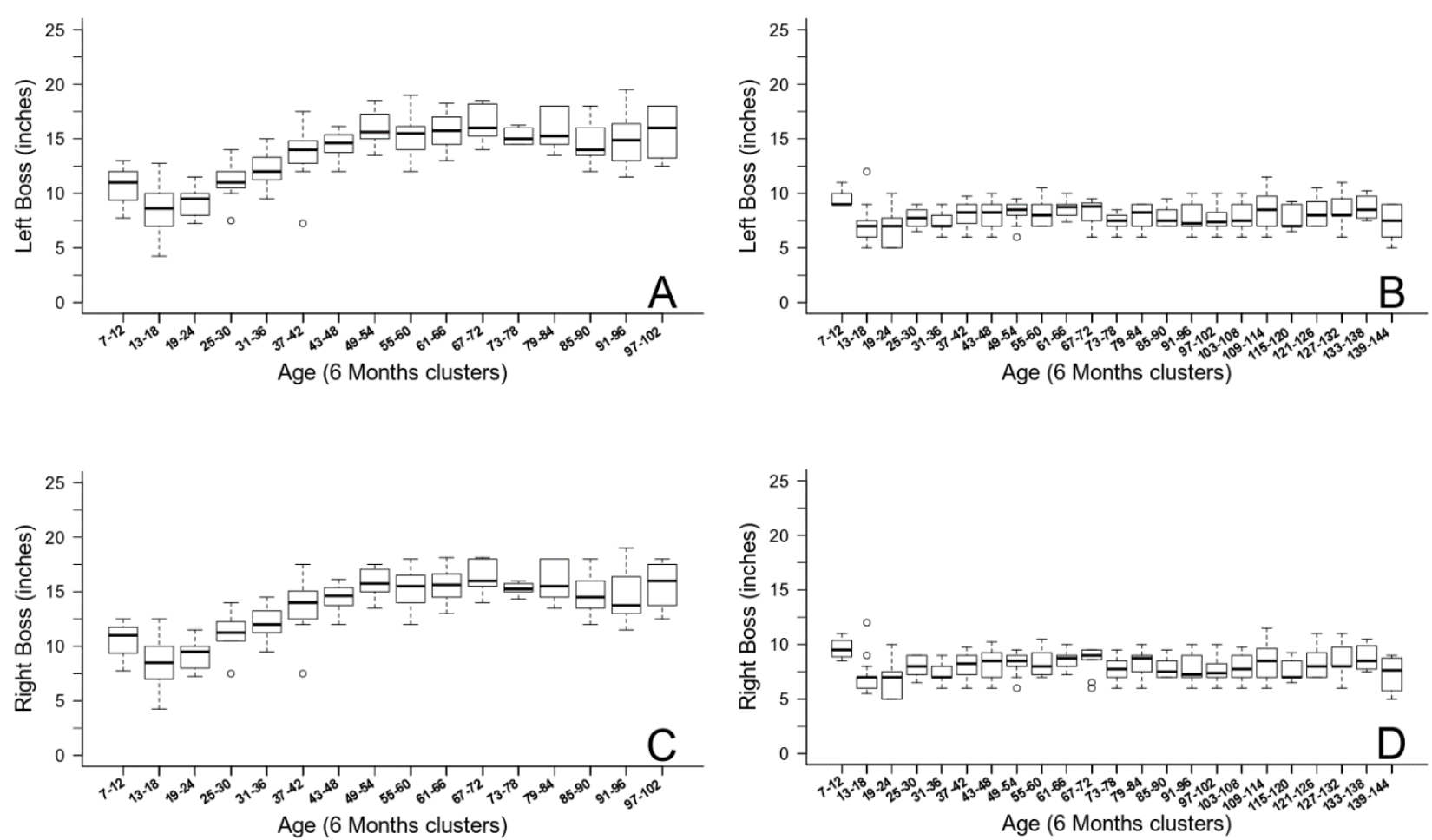

Figure 4 Horn boss distribution (BOSL-BOSR) for male ( $A$ and $C$ ) and female (B and $D)$ clustered within six months

Table 2 Posterior estimates of variance components and heritability estimates $\left(\mathrm{h}^{2}\right)$ with their associated posterior standard deviations (SD) for economic important horn traits

\begin{tabular}{lcccc}
\hline Trait & $\sigma_{a}^{2}(\mathrm{SD})$ & $\sigma_{p e}^{2}(\mathrm{SD})$ & $\sigma_{e}^{2}(\mathrm{SD})$ & $\boldsymbol{h}^{2}(\mathrm{SD})$ \\
\hline BHSO & $3.057(0.970)$ & $3.006(1.067)$ & $10.138(0.753)$ & $0.188(0.057)$ \\
BHTSCl & $1.344(1.328)$ & $30.008(5.141)$ & $35.314(3.481)$ & $0.020(0.019)$ \\
BOSL & $0.394(0.268)$ & $1.222(0.367)$ & $2.007(0.233)$ & $0.108(0.071)$ \\
BOSR & $0.398(0.266)$ & $1.217(0.360)$ & $1.842(0.219)$ & $0.115(0.074)$
\end{tabular}

BHSO = Horn spread; bhtsci $=$ Tip to tip index; $\mathrm{BOSL}=$ boss left measurement; $\mathrm{BOSR}=$ boss right measurement; $\sigma_{a}^{2}=$ additive genetic variance; $\sigma_{p e}^{2}=$ permanent environmental variance; $\sigma_{e}^{2}=$ residual error variance

Regarding the current correlations (Table 3 ) it is clear that, phenotypically, there is a close relationship between traits (over 0.7). However, genetically the only close association is between left and right boss (BOSL-BOSR). Therefore, selecting for horn length or spread based on any other evaluated traits will not effectively contribute to its development and, even between these two (BHSO-BHTSCl), could cause a decrease in growth as they have a negative genetic correlation.

The average inbreeding coefficient based on genomic verified pedigrees for animals born in 2015 was 0.008 , which is in line with the low inbreeding results of molecular studies done by Van der Westhuizen (2016) on Cape buffalo. These results suggest that the breeding management of the studied Cape buffalo population has not diminished their genetic variance. 
Table 3 Posterior genetic correlations and posterior standard deviations (SD) (above diagonal) and phenotypic correlations with standard errors (SE) (below diagonal) for buffalo horns traits

\begin{tabular}{lcccc}
\hline & BHSO & BHTSCI & BOSL & BOSR \\
\hline BHSO & & $-0.203(0.617)$ & $0.382(0.389)$ & $0.397(0.383)$ \\
BHTSCI & $0.889(0.023)$ & & $0.041(0.549)$ & $0.037(0.550)$ \\
BOSL & $0.712(0.036)$ & $0.777(0.039)$ & & $0.900(0.075)$ \\
BOSR & $0.711(0.036)$ & $0.776(0.039)$ & $0.997(0.004)$ &
\end{tabular}

$\overline{\mathrm{BHSO}}=$ Horn spread; $\mathrm{BHTSCI}=$ Tip to tip index; $\mathrm{BOSL}=$ boss left measurement; $\mathrm{BOSR}=$ boss right measurement

\section{Conclusions and Recommendations}

Based on the results of the current study it is not economically viable to measure the tip to tip horn spread of females after 43 - 48 months of age. The genetic and statistical evaluations indicated that the boss measurements for females and young animals are unreliable which is thought to be partly attributed to the measuring technique. It is inaccurate to compare differences between the left and right boss size, as only one side is often measured, and it is assumed (usually) and recorded the other side as the same. The inbreeding coefficient suggests that breeding management of the studied Cape buffalo population retained adequate genetic diversity, which corresponds with previous population studies. But it has to be highlighted that special attention has to be given to reproduction and inbreeding if a breeding programme is implemented, as inbreeding could become critical if selection is based exclusively on horn traits. Based on the genetic correlations and whether they are accurate, it cannot be assumed that by selecting for one trait will necessarily improve another horn trait (except the left and right bosses). It is recommended that a standard recording and identification system for buffalo should be implemented. Further genetic evaluation studies and the development of growth curves using repeated measurements for the same animals throughout their lifetime are needed to support the results of the current study. Linear traits (e.g. scores 1 - 9) for horn shape and/or an index combining the SCl and RW (e.g. by subtracting the latter from the former to give an indication of the horn shape) should be developed. Horn stress tests should be conducted to establish whether horn shape will affect the strength of the horn. Finally, additional studies are required to identify the causes of non-typical horn dimensions based on sex (e.g. fertility).

\section{Acknowledgements} evaluation.

The authors would like to acknowledge Wildlife Stud Services for providing the Cape buffalo dataset for its

\section{Authors' contributions}

AAL designed the experiment and analysed the data. GCJ and AAL structured the scientific content and drafted the manuscript. FWCN and JBVW assisted with the statistical analysis. All authors contributed in the editorial content.

\section{Conflict of interest declaration}

The authors declare that they have no conflict of interests regarding the present study.

\section{References}

Alden, P.C., Estes, R.D., Schlitter, D. \& McBride, B., 1995. National Audubon Society Field Guide to African Wildlife. Knopf, New York.

Cloete, P.C., Van der Merwe, P. \& Saayman, M., 2015. Economic overview of SA wildlife industry. Chapter 4. In Game ranch profitability in South Africa. 2nd ed. ABSA, Cape Town. pp. 63-79

Falconer, D.S. \& Mackay, T.F.C., 1996. Introduction to Quantitative Genetics. 4th ed. Longman, Harlow, Essex, UK.

Greyling, B.J., 2007. Genetic variation, structure and dispersal among Cape buffalo populations from the HluhluweImfolozi and Kruger National Parks of South Africa. Unpublished PhD Thesis. University of Pretoria, Department of Zoology and Entomology, Pretoria, South Africa. $175 \mathrm{p}$.

Greyling, B.J., Kryger, P., Du Plessis, S., Van Hooft, W.F., Van Helden, P., Getz, W.M. \& Bastos, A.D.S., 2008. Development of a high-throughput microsatellite typing approach for forensic and population genetic analysis of wild and domestic African Bovini. Afr. J. Biotechnol. 7, 655-660.

Hadfield, J.D., 2010. MCMC Methods for Multi-Response Generalized Linear Mixed Models: The MCMCgImm R Package. J. Stat. Softw. 33, 1-22.

Hadfield, J.D., 2017. MCMCglmm course notes. CRAN - Package MCMCglmm. From: https://cran.rproject.org/web/packages/MCMCglmm/index.html (Accessed 9 November 2017). 
Johnston, S.E., Beraldi, D., McRae, A.F., Pemberton, J.M. \& Slate, J., 2009. Horn type and horn length genes map to the same chromosomal region in Soay sheep. Heredity 104, 196-205.

Lepori, A.A., Josling, G.C., Neser, F.W.C., Lubout, P.C. \& Van Wyk, J.B., 2018. Preliminary results evaluating horn traits of economic importance in the Cape buffalo (Syncerus caffer caffer). In: Proceedings of the 11th World Congress on Genetics Applied to Livestock Production. Auckland, New Zealand. p. 530.

Mrode, R., 2006. Linear Models for the Prediction of Animal Breeding Values. 2nd ed. CABI, Oxfordshire, UK.

Pigeon, G., Festa-Bianchet, M., Coltman, D.W. \& Pelletier, F., 2016. Intense selective hunting leads to artificial evolution in horn size. From: https://doi.org/10.1111/eva.12358. Evol. Appl. 9, 521-530.

Poissant, J., Wilson, A.J., Festa-Bianchet, M., Hogg, J.T. \& Coltman, D.W., 2008. Quantitative genetics and sex-specific selection on sexually dimorphic traits in bighorn sheep. Proc. R. Soc. B. Biol. Sci. 275, 623-628.

R Core Team., 2017. R: A Language and Environment for Statistical Computing. R Foundation for Statistical Computing, Vienna, Austria.

Simpson, I.F., 2012. The development of organised game ranching in South Africa. Chapter 6. In Mpunzi, dawn of a new era: An historical overview of the development of the game ranching industry in Kwazulu-Natal and Southern Africa in the 20th century. Cape Town. pp. 83-90

Taylor, A., Lindsey, P.A., Davies-Mostert, H. \& Goodman, P., 2015. An assessment of the economic, social and conservation value of the wildlife ranching industry and its potential to support the green economy in South Africa. Johannesburg. South Afr. Endanger. Wildl. Trust.

Van der Westhuizen, L., Neser, F.W.C. \& Roan, L., 2016. Genetic variability of Cape buffalo populations in South Africa. In 9th International Wildlife Ranching Symposium (IWRS) \& IUCN 2nd African Buffalo Symposium. Windhoek, Namibia.

Van Rooyen, N., Van Rooyen, J. \& Van Rooyen, N., 2016. Handling and measuring trophies. In: Game Ranch Management. Bothma, J.duP. \& Du Toit, J.G., eds. 6th ed. Van Schaik, Pretoria. pp. 715-753

Wilson, A.J., Réale, D., Clements, M.N., Morrissey, M.M., Postma, E., Walling, C.A., Kruuk, L.E.B. \& Nussey, D.H., 2010. An ecologist's guide to the animal model. J. Anim. Ecol., 79, 13-26. From: https://doi.org/10.1111/j.13652656.2009.01639.x 